\title{
Factores no genéticos en el crecimiento predestete de corderos biotipo lana en el trópico de altura colombiano
}

\author{
Non-genetic factors in pre-weaning growth performance of wool biotype \\ lambs in the Colombian highland tropics \\ María Alejandra Cuello Estrada ${ }^{1}$, José David Moreno Torres ${ }^{1}$, Fredy Armado \\ Aguilar Aguilar², Andrea Baracaldo Martínez ${ }^{2}$, Jorge Eduardo Atuesta-Bustos ${ }^{2,3}$
}

\section{Resumen}

El estudio tuvo como objetivo evaluar el efecto de factores no genéticos sobre las características del crecimiento predestete en ovinos bajo condiciones tropicales. Se evaluó el sexo de la cría, el tipo de nacimiento y el número de partos de la madre sobre los pesos al nacimiento, pesos al destete y la ganancia diaria predestete de 52 corderos biotipo lana. Las crías fueron pesadas semanalmente desde el nacimiento hasta la semana 12 (destete). El sexo de la cría no tuvo un efecto significativo sobre las variables evaluadas. Los corderos provenientes de madres de tercer parto presentaron una mayor ganancia diaria predestete que los corderos provenientes de ovejas de primer parto $(\mathrm{p}<0.05)$. Los corderos provenientes de partos simples fueron más pesados al nacimiento y al destete que corderos provenientes de partos múltiples $(\mathrm{p}<0.05)$. Se concluye que el tipo de nacimiento es el factor que principalmente afecta las características de crecimiento predestete y que el sexo de la cría y el número de partos no afectan significativamente las características en mención.

Palabras clave: destete; ovinos; sexo; tipo de nacimiento; número de partos; trópico

\section{AbstraCt}

The objective of this study was to evaluate the effect of non-genetic factors on the characteristics of pre-weaning growth in sheep under tropical conditions. The sex of the lamb, the type of birth (single, multiple) and parity number on the birth weight, weaning weight and preweaning daily gain of 52 wool biotype lambs were evaluated. The lambs

\footnotetext{
${ }^{1}$ Semillero de Investigación en Ciencias Animales UNIAGRARIA, Facultad de Ciencias Agrarias, Fundación Universitaria Agraria de Colombia, Colombia

${ }^{2}$ Facultad de Ciencias Agrarias, Fundación Universitaria Agraria de Colombia, Colombia

${ }^{3}$ E-mail: atuesta.jorge@uniagraria.edu.co
} 
were weighed weekly from birth to week 12 (weaning). The sex of lambs did not have a significant effect on the variables evaluated. Lambs from mothers of third lambing had a higher preweaning daily gain than lambs from mothers of first lambing $(p<0.05)$. Singleborn were heavier at birth and at weaning than lambs born as twins ( $\mathrm{p}<0.05)$. It is concluded that the type of birth is the factor that mainly affects the characteristics of preweaning growth and that the sex of the offspring and lambing number do not significantly affect the growth performance.

Palabras clave: weaning; sheep; sex; type of birth; parity number; tropics

\section{INTRODUCCIÓN}

El crecimiento en los corderos desde el nacimiento hasta el momento del destete se encuentra afectado por factores genéticos (raza de los padres) y no genéticos (ambientales) entre los que se incluyen la edad de la madre (número de partos), el estado nutricional durante la gestación y la lactancia, el tipo de parto, sexo de la cría y estado sanitario (Basáñez, 2014; Petrovic et al., 2015a).

En este sentido, ha sido planteado que el sexo de la cría y el número de corderos nacidos en cada parto son variables que influyen en el crecimiento de los ovinos y que merecen ser evaluadas, ya que estas características también se encuentran fuertemente relacionadas con la rentabilidad del sistema productivo, que en el caso de la producción de carne se busca generar animales más pesados en periodos más cortos de tiempo (Macedo y Arredondo, 2008; Moyano et al., 2017). Así mismo, se reconoce que el número de partos de la oveja tiene influencia sobre el tamaño de camada, mortalidad perinatal, peso al nacimiento, peso al destete y tasa de crecimiento postnatal (Macedo y Humel, 2006), por lo que convendría evaluar los cambios de peso de los corderos como indicador de productividad de la oveja durante la lactancia (García-Osorio et al., 2017).

Desde la perspectiva del productor, el conocimiento de características como el peso al nacimiento, la ganancia diaria de peso predestete y el peso al destete les permite un manejo más eficiente del proceso de selección de los reemplazos, así como reconocer la eficiencia productiva del rebaño (HinojosaCuellar et al., 2012). No obstante lo anterior y a pesar de la importancia de la evaluación de estos factores, el número de estudios relacionados con la influencia de factores no genéticos sobre las características de crecimiento en ovinos bajo condiciones tropicales son escasos (Mellado et al., 2016; OlivaHernández y García-Osorio, 2016). Por otro lado, se reconoce que puede haber variaciones particulares por raza o sistema de producción con relación al efecto del sexo, tipo de parto y número de partos sobre el crecimiento de los ovinos, lo que requiere estudios in situ en condiciones de trópico (Macedo y Arredondo, 2008).

Con base en lo anterior, el objetivo de este estudio fue evaluar el efecto del sexo de la cría, el número de crías por parto y el número de partos sobre el crecimiento predestete en ovinos de lana bajo condiciones del trópico de altura colombiano.

\section{Materiales y Métodos}

El estudio se realizó en las instalaciones de la Finca Santa María, perteneciente a la Universidad La Gran Colombia, situada a una altitud de $2562 \mathrm{msnm}$, en el municipio de Chía, departamento de Cundinamarca, Colombia. La zona presenta una temperatura promedio de $14{ }^{\circ} \mathrm{C}$ y una precipitación media de 750 
mm. El tipo de clima es Af (selva tropical), usando la clasificación de Köppen.

Se utilizaron 52 crías provenientes de diferentes cruces de ovinos biotipo lana (Romney Marsh, Ile de France y Moro Colombiano), siendo 31 machos y 21 hembras. El tamaño muestral fue por conveniencia al incluir los individuos (crías) disponibles en la finca en el momento de realizar el estudio (Segura, 2000). Las crías provenían de madres de primer $(n=3)$, segundo $(n=4)$, tercer $(n=16)$ y cuarto parto $(n=13)$. Asimismo, los corderos provenían de madres de parto simple $(n=20)$ y de parto múltiple $(n=16)$.

Los corderos fueron pesados semanalmente desde el nacimiento (semana 0 ) hasta los tres (3) meses de edad (semana 12), en que fueron destetados. El pesaje de los animales se hizo en las mañanas, mediante una báscula con capacidad de hasta $150 \mathrm{~kg}$ y con resolución de $0.02 \mathrm{~kg}$.

Las crías fueron mantenidas en corrales independientes junto con sus madres, las cuales recibieron durante los primeros siete días una dieta basada en un suplemento de maíz quebrado, alfalfa peletizada comercial, alimento balanceado comercial para cerdos en finalización, en proporción 1:1:1 en base húmeda, la cual fue ofertada a razón de 350 $\mathrm{g} /$ día y heno a voluntad. Luego de este tiempo, las hembras fueron llevadas con sus crías a áreas de pastoreo con forraje kikuyo (Pennisetum clandestinum) en un sistema de pastoreo continuo.

Los datos del desempeño del crecimiento predestete fueron analizados mediante análisis de varianza con medidas repetidas en el tiempo utilizando el procedimiento MIXED del paquete estadístico SAS University, 2016®. El modelo consideró como efectos fijos al tipo de nacimiento (parto simple, parto múltiple), el sexo de las crías, la semana de pesaje y sus interacciones. El número de partos de la hembra fue considerado como una covariable dentro del modelo. Las medias fueron comparadas con la prueba de Tukey-Kramer (á=0.05).
El modelo estadístico empleado fue Yijkl $=\beta 0+\beta 1 \mathrm{xijk} \mathrm{l}+\rho \mathrm{j}+\tau \mathrm{k}+\mathrm{tl}+\delta \mathrm{ijk}+\left(\rho^{*} \tau\right) \mathrm{jk}+$ $\left(\rho^{*} \mathrm{t}\right) \mathrm{jl}+\left(\tau^{*} \mathrm{t}\right) \mathrm{kl}+\left(\rho^{*} \tau^{*} \mathrm{t}\right) \mathrm{jkl}+\varepsilon \mathrm{ijkl}$, donde Yijkl $=$ Peso corporal para el i-ésimo individuo, del j-ésimo sexo, del del k-+ésimo tipo de parto, en la l-esima semana; $\beta 0=$ Intercepto; $\beta 1=$ Coeficiente de la regresión; $x i j k l=$ Número de parto de la madre; $\rho j$ = Efecto del sexo j; $\tau \mathrm{k}=$ Efecto del tipo de parto $\mathrm{k} ; \mathrm{tl}=$ Efecto de la semana $1 ; \delta \mathrm{ijk}=$ Error aleatorio con media 0 y varianza $\sigma 2$ (varianza entre animales dentro de un mismo tratamiento; es decir, para cada combinación de sexo por tipo de parto); $\left(\rho^{*} \tau\right) \mathrm{jk}=$ Efecto de la interacción del sexo $\mathrm{j}$ con el tipo de parto $\mathrm{k} ;\left(\rho^{*} \mathrm{t}\right) \mathrm{jl}=$ Efecto de la interacción del sexo j con la semana $1 ;\left(\tau^{*} \mathrm{t}\right) \mathrm{kl}$ $=$ Efecto de la interacción del tipo de parto $\mathrm{k}$ con la semana $1 ;\left(\rho^{*} \tau^{*} t\right) j \mathrm{kl}=$ Efecto de la interacción del sexo $\mathrm{j}$ con el tipo de parto $\mathrm{k} y$ la semana 1; $\varepsilon \mathrm{ijkl}=$ Error aleatorio con media 0 y varianza $\sigma 2$ (varianza entre los peso sucesivos en los animales); $\mathrm{i}=$ número de individuos; $j=$ tipo de sexo del animal: $\mathrm{k}=$ tipo de parto; $1=$ semana de pesaje.

\section{Resultados y Discusión}

\section{Efecto del Sexo de la Cría}

El sexo del cordero no afectó el peso al nacimiento (Cuadro 1), tal y como ha sido reportado en biotipos de pelo (Quintero et al., 1997; Hinojosa-Cuellar et al., 2012) y en biotipos de lana (Basañez, 2014; GonzálezAnaya et al., 2013; Norouzian, 2015). No obstante, se tienen reportes que indican un mayor peso de los machos al nacimiento en comparación con las hembras (Lupi et al., 2015; Petrovic et al., 2015b; Zidane et al., 2015; Mirderikvandi et al., 2016).

Se reconoce que los machos presentan mayores tasas de crecimiento respecto a las hembras a nivel uterino, debido a la presencia del cromosoma $Y$ y a la activación de los productos del gen sry, como los andrógenos y la hormona antimulleriana (Gardner et al., 2007). Así mismo, si bien el número de 
Cuadro 1. Medias ( \pm error estándar) por mínimos cuadrados del peso al nacimiento, peso al destete y ganancia diaria de peso afectado por el sexo de la cría, número de partos y la interacción tipo de nacimiento por edad de la cría

\begin{tabular}{lccc}
\hline Factor & $\begin{array}{c}\text { Peso al } \\
\text { nacimiento }(\mathrm{kg})\end{array}$ & $\begin{array}{c}\text { Peso al destete } \\
(\mathrm{kg})\end{array}$ & $\begin{array}{c}\text { Ganancia diaria } \\
\text { de peso }(\mathrm{g} / \mathrm{d} \text { ía })\end{array}$ \\
\hline Sexo & n.s. & n.s. & n.s. \\
$\quad$ Macho $(\mathrm{n}=21)$ & $3.76 \pm 0.21$ & $14.88 \pm 0.65$ & $131.5 \pm 8.0$ \\
$\quad$ Hembra $(\mathrm{n}=31)$ & $3.56 \pm 0.19$ & $14.88 \pm 0.70$ & $135.4 \pm 7.4$ \\
$\begin{array}{l}\text { Tipo de nacimiento x edad } \\
\text { de la cría (en semanas) }\end{array}$ & $*$ & $*$ & - \\
N. ${ }^{\circ}$ de partos de la madre & & & $*$ \\
1 (n=3) & n.s. & n.s. & $89 \pm 17^{\mathrm{b}}$ \\
$2(\mathrm{n}=4)$ & $3.0 \pm 0.41$ & $10.5 \pm 1.46$ & $112 \pm 18^{\mathrm{ab}}$ \\
$3(\mathrm{n}=16)$ & $4.4 \pm 0.43$ & $12.7 \pm 1.54$ & $144 \pm 7^{\mathrm{a}}$ \\
$4(\mathrm{n}=13)$ & $4.3 \pm 0.21$ & $15.5 \pm 0.63$ & $135 \pm 9^{\mathrm{ab}}$ \\
\hline
\end{tabular}

n.s.: efecto no significativo; *: $p<0.05 ;$-: no estudiado

${ }^{a b}$ Medias con diferentes literales dentro de la columna indican diferencias significativas ( $p$ ?0.05)

Cuadro 2. Medias ( \pm error estándar) por mínimos cuadrados para el peso corporal del cordero y su relación con el tipo de nacimiento (simple $[\mathrm{n}=20]$, múltiple $[\mathrm{n}=16]$ ) y la edad de la cría

\begin{tabular}{|c|c|c|c|}
\hline \multirow{2}{*}{$\begin{array}{l}\text { Edad de la cría } \\
\text { (semanas) }\end{array}$} & \multicolumn{2}{|c|}{ Tipo de nacimiento } & \multirow[b]{2}{*}{ Valor P } \\
\hline & $\begin{array}{l}\text { Madres de parto simple } \\
(\mathrm{kg})\end{array}$ & $\begin{array}{l}\text { Madres de parto múltiple } \\
\qquad(\mathrm{kg})\end{array}$ & \\
\hline 0 & $4.23 \pm 0.23$ & $3.06 \pm 0.16$ & $0.0286^{*}$ \\
\hline 1 & $5.07 \pm 0.28$ & $3.82 \pm 0.20$ & 0.1225 \\
\hline 2 & $6.04 \pm 0.33$ & $4.49 \pm 0.23$ & 0.0781 \\
\hline 3 & $7.26 \pm 0.36$ & $5.24 \pm 0.25$ & $0.0115^{*}$ \\
\hline 4 & $8.31 \pm 0.41$ & $5.99 \pm 0.28$ & $0.0075^{*}$ \\
\hline 5 & $9.48 \pm 0.45$ & $6.75 \pm 0.31$ & $0.0029 *$ \\
\hline 6 & $10.66 \pm 0.49$ & $7.55 \pm 0.34$ & $0.0016^{*}$ \\
\hline 7 & $11.75 \pm 0.51$ & $8.56 \pm 0.361$ & $0.0022 *$ \\
\hline 8 & $12.86 \pm 0.59$ & $9.32 \pm 0.41$ & $0.0039^{*}$ \\
\hline 9 & $13.86 \pm 0.64$ & $10.21 \pm 0.45$ & $0.0077^{*}$ \\
\hline 10 & $14.91 \pm 0.70$ & $11.01 \pm 0.49$ & $0.0094 *$ \\
\hline 11 & $15.96 \pm 0.74$ & $11.86 \pm 0.51$ & $0.0104 *$ \\
\hline 12 & $17.01 \pm 0.78$ & $12.74 \pm 0.54$ & $0.012 *$ \\
\hline
\end{tabular}


placentomas fetales no difiere entre sexos, el peso del placentoma asociado a los machos es $10.5 \%$ superior al de las hembras, lo que puede suponer una mayor captación de nutrientes y conllevar a mayores pesos al nacimiento (Macedo y Arredondo, 2008).

En este estudio tampoco se encontró un efecto significativo del sexo de la cría sobre el peso al destete ni la ganancia diaria de peso predestete (Cuadro 1); resultados que difieren con lo expuesto por Yilmaz et al. (2007) en corderos Norduz, donde los machos fueron más pesados que las hembras. En este mismo sentido, Hinojosa-Cuellar et al. (2009), trabajando con biotipos de pelo, reportaron diferencias significativas de 5.6 y $7.8 \%$ para las variables peso al destete y ganancia diaria de peso predestete a favor de los machos. Ramírez-Tello et al. (2013), reportan en forma similar, mayores pesos al destete y mayor ganancia diaria de peso predestete en machos.

En contraste, otros autores concuerdan con los resultados del presente estudio, indicando pesos similares al destete y en la ganancia diaria de peso predestete entre machos y hembras (Benyi et al., 2006; Koritiaki et al., 2013; González-Domínguez et al., 2016), e incluso, Gbangboche et al. (2006) reportaron mayores tasas de ganancia diaria de peso entre los días 30 y 60 días de edad para las hembras.

El efecto hormonal de la testosterona que promueve un mayor crecimiento en los machos no se manifiesta hasta después de los 70 días de nacimiento, momento en que se realiza el destete (Bores et al., 2002; Hinojosa-Cuellar et al., 2012), lo que puede explicar parcialmente el comportamiento similar entre sexos en este estudio.

\section{Efecto del Número de Partos}

El número de partos de la oveja no afectó el peso al nacimiento ni el peso al destete de los corderos (Cuadro 1). Estos resultados son similares a otros reportes (Aktas y Dogan,
2014; Karakus y Atmaca, 2016; Yilmaz et al., 2018). No obstante lo anterior, se pudo apreciar una tendencia a un mayor peso al nacimiento y al destete con el incremento del número de parto de la madre, lo cual coincide con Taye et al. (2010), Hussain et al. (2013) y Aktas et al. (2015), quienes indican que ovejas de mayor edad o mayor número de partos producen corderos más pesados.

El efecto de la edad de la madre sobre el peso corporal de los corderos puede ser explicado por tres factores. Primero, la producción de leche y la habilidad materna se incrementa con la edad de la madre; segundo, la variación en tamaño y el imprinting fisiológico del útero durante la primera gestación facilitará el mayor crecimiento fetal en posteriores preñeces; y tercero, las ovejas jóvenes utilizan la energía disponible para crecimiento y desarrollo corporal, mientras que ovejas maduras utilizan esta energía para el desarrollo del cordero (Gardner, 2007; Taye et al., 2010; Baneh et al., 2013).

Las ganancias diarias de peso predestete fueron mayores $(p<0.05)$ en corderos provenientes de ovejas de tercer parto en comparación con ovejas de primer parto. Teklebrhan et al. (2014), por otro lado, encontraron una menor ganancia diaria de peso en corderos después del tercer parto de las ovejas, posiblemente por una disminución de la producción de leche de las madres.

\section{Efecto del Tipo de Nacimiento del Cor- dero}

El tipo de nacimiento (simple o múltiple) y la edad del cordero presentaron una interacción estadísticamente significativa $(\mathrm{p}<0.05)$, de allí que los resultados fueron analizados de manera conjunta. El tipo de nacimiento influenció los pesos corporales de los corderos de manera consistente (Cuadro 2). Así mismo, los corderos provenientes de partos simples fueron significativamente más pesados al nacimiento (semana 0 ) comparados con aquellos provenientes de partos múltiples $(\mathrm{p}<0.05)$. Este mismo resultado se ob- 
servó en varias semanas, incluyendo la semana del destete (semana 12), tal y como se describe en la literatura (Chniter et al., 2009; Yilmazy Altin, 2011; González-Domínguez et al., 2016; Deribe et al., 2017).

Se reconoce, que aunque el peso de la placenta y el número de placentomas se incrementa en ovejas que gestan más de una cría, los corderos provenientes de nacimientos dobles y triples se encuentran asociados a un menor número de placentomas comparados con corderos de parto simple (Gootwine, 2005), lo que puede explicar los menores pesos al nacimiento. De igual manera, el área de superficie de intercambio de cada placentoma se reduce con el incremento en el tamaño de camada, lo que resulta en un menor aporte de nutrientes para los fetos, especialmente en la fase de crecimiento exponencial al final de la preñez (Dwyer $e t$ al., 2005).

En este mismo contexto, los corderos provenientes de partos simples presentan mayores tasas de crecimiento y a su vez mayores pesos al destete comparados con corderos nacidos de partos múltiples (Mellado et al., 2016; Yilmaz et al., 2018); resultados que coinciden con los obtenidos en este estudio. Se reconoce que el consumo de energía durante la gestación y lactancia de ovejas con gestaciones múltiples no es suficiente para permitir a sus corderos alcanzar su potencial de crecimiento; de igual manera, los corderos provenientes de partos simples reciben más leche de sus madres $y$, por lo tanto, tienen una mayor tasa de crecimiento y mayores pesos al destete, cuando son comparados con corderos provenientes de nacimientos dobles y triples (Gabr et al., 2016).

\section{Conclusiones}

- El sexo de los corderos no tuvo un efecto significativo sobre el peso al nacimiento, la ganancia diaria de peso predestete $\mathrm{y}$ el peso al destete.
- El número del parto de las hembras no tuvo un efecto significativo sobre el peso al nacimiento y el peso al destete.

- Los corderos provenientes de madres de tercer parto presentaron mayor ganancia diaria de peso predestete que los corderos provenientes de ovejas de primer parto $(\mathrm{p}<0.05)$.

- Se presentó una interacción significativa $(p<0.05)$ entre el tipo de nacimiento (simple vs. múltiple) y la edad en semanas del cordero, siendo mayores los pesos de crías provenientes de partos simples.

\section{Agradecimiento}

Los autores agradecen a la Universidad La Gran Colombia y al Sr. Iván Acevedo por permitir la realización del experimento en las instalaciones de la granja ovina Santa María.

\section{Literatura Citada}

1. Aktas AH, Dogan '. 2014. Effect of live weight and age of Akkaraman ewes at mating on multiple birth rate, growth traits, and survival rate of lambs. Turk $\mathrm{J}$ Vet Anim Sci 38: 176-182. doi: 10.3906/ vet-1301-10

2. Aktas AH, Dursun S, Dogan P, Kiyma Z, Demirci U, Halici Ý. 2015. Effects of ewe live weight and age on reproductive performance, lamb growth, and survival in Central Anatolian Merino sheep. Arch Anim Breed 58: 451-459. doi: 10.5194/aab-58-451-2015

3. Baneh H, Rokouei M, Ghafouri-Kesbi F, Veysi A, Niknafs S. 2013. Multivariate genetic analysis on body weight traits in Ghezel sheep. Songklanakarin J Sci Technol 35: 131-135.

4. Basáñez CJ. 2014. Producción de corderos y efecto del sexo y tipo de parto en ovejas Polypay en sistema estabulado. Tesis de Ingeniero Agrónomo Zootecnista. México: Univ Autónoma de San Luis Potosí. 28 p. 
5. Benyi K, Norris D, Karbo N, Kgomo KA. 2006. Effects of genetic and environmental factors on pre-weaning and post-weaning growth in West African crossbred sheep. Trop Anim Health Prod 38: 547-554. doi: 10.1007/s11250-006-4416-2

6. Bores RF, Velázquez PA, Heredia M. 2002. Evaluación de razas terminales en esquemas de cruza comercial con ovejas de pelo F1. Téc Pecu Mex 40: 71-79.

7. Chniter M, Maali S, Hammadi M, Khorchani T, Harab H, Krit R, Ben Hamouda M, et al. 2009. Effects of dam age, litter size and gender on birth weight of D'man lamb: consequence on lamb mortality. J Arid Land Stud 19: 169-172.

8. Deribe G, Girma A, Gebeyehu G, Asrat T, Belete SG. 2017. Early growth and survival rates of crossbred lambs (Dorper $\mathrm{x}$ indigenous) under semiintensive management at Areka, Southern Ethiopia: effects of non-genetic factors. Afr J Agric Res 12: 2008-2016. doi: 10.5897/AJAR2017.12280

9. Dwyer CM, Calvert SK, Farish M, Donbavand J, Pickup HE. 2005. Breed, litter and parity effects on placental weight and placentome number, and consequences for the neonatal behaviour of the lamb. Theriogenology 63: 1092-1110. doi: 10.1016/j.theriogenology.2004.06.003

10. Gabr AA, Shalaby NA, Ahmed ME. 2016. Effect of ewe born type, growth rate and weight at conception on the ewe subsequent productivity of Rahmani sheep. Asian J Anim Vet Adv 11: $732-$ 736. doi: 10.3923/ajava.2016.732.736

11. García-Osorio IC, Oliva-Hernández J, Osorio-Arce MM, Torres-Hernández G, Hinojosa-Cuéllar JA, GonzálezGarduño R. 2017. Influencia materna en el crecimiento predestete y características de la canal de corderos de pelo. Ecosistemas Recur Agropecu 4: 51-63. doi: 10.19136/era.a4n10.818.

12. Gardner DS, Buttery PJ, Daniel Z, Symonds ME. 2007. Factors affecting birth weight in sheep: Maternal environment. Reproduction 133: 297-307. doi: 10.1530/REP-06-0042
13. Gbangboche AB, Youssao AKI, Senou M, Adamou-Ndiaye M, Ahissou A, Farnir F, et al. 2006. Examination of non-genetic factors affecting the growth performance of Djallonke sheep in Soudanian zone at the Okpara breeding farm of Benin. Trop Anim Health Pro 38: 55-64. doi: 10.1007/s11250-006-4231-9

14. González-Anaya A, Ochoa-Cordero MA, Torres-Hernández G, DíazGómez MO, González-Camacho JM. 2013. Influencia de factores ambientales en el comportamiento productivo en fase predestete de corderos Rambouillet. Abanico Vet 3: 31-38.

15. González-Domínguez, G, HinojosaCuéllar JA, Oliva-Hernández, J, Torres-Hernández G, Segura-Correa $J C$, González-Garduño R, GarcíaOsorio IDC. 2016. Análisis del crecimiento predestete de corderos Barbados Barriga Negra en clima cálido húmedo. Nova Scientia 8: 181-197.

16. Gootwine E. 2005. Variability in the rate of decline in birth weight as litter size increases in sheep. Anim Sci 81: 393398. doi: 10.1079/ASC41160393

17. Hinojosa-Cuéllar J, Oliva-Hernández J, Torres-Hernández G, Segura-Correa J, Aranda-Ibáñez E, GonzálezCamacho J. 2012. Factores que afectan el crecimiento predestete de corderos Pelibuey en el trópico húmedo de México. Univ Cienc Trópico Húmedo 28: 163-171.

18. Hinojosa-Cuéllar JA, RegaladoArrazola F, Oliva-Hernández J. 2009. Crecimiento prenatal y predestete en corderos Pelibuey, Dorper, Katahdin y sus cruces en el sureste de México. Rev Cient 19: 522-532.

19. Hussain A, Akhtar P, Ali S, Younas M, Yaqoob M, Babar ME, Javed K, Shakoor A. 2013. Factors influencing body weights at different ages in Thalli sheep. J Anim Plant Sci 23: 1-6.

20. Karakus F, Atmaca M. 2016. The effect of ewe body condition at lambing on growth of lambs and colostral specific gravity. Arch Anim Breed 59: 107-112. doi: 10.5194/aab-59-107-2016 
21. Koritiaki NA, Ribeiro DA, Mizubuti IY, Silva LD, Barbosa MA, Bumbieris $V H$, et al. 2013. Influence of environmental factors on ponderal performance and morphometric characteristics of lambs of different genetic groups from birth to weaning. Rev Bras Zootec 42: 463-470. doi: 10.1590/S151635982013000700001

22. Lupi TM, Nogales S, León JM, Barba C, Delgado J V. 2015. Analysis of the non-genetic factors affecting the growth of Segureño sheep. Ital J Anim Sci 14: 124-131. doi: 10.4081/ ijas.2015.3683

23. Macedo R, Arredondo V. 2008. Efecto del sexo, tipo de nacimiento y lactancia sobre el crecimiento de ovinos Pelibuey en manejo intensivo. Arch Zootec 57: 219-228.

24. Macedo R, Hummel JD. 2006. Influence of parity on productive performance of Pelibuey ewes under intensive management in the Mexican dry tropics. Livestock Res Rural Develop18(6). [Internet]. Disponible en: http:// www.lrrd.org/lrrd18/6/mace18077.htm

25. Mellado J, Marín V, Reyes-Carrillo JL., Mellado M, Gaytán L, De Santiago M. 2016. Effects of non-genetic factors on preweaning growth traits in Dorper sheep managed intensively in central Mexico. Ecosistemas Recur Agropecu 3: 229-235.

26. Mirderikvandi M, Masoudi A, Khaldari M, Bojarpour M, Nazari, $P$. 2016. The effect of some factors on growth performance of Lori Bakhtiari lambs. Livestock Res Rural Develop 28(12). [Internet]. Disponible en: http:// www.lrrd.org/lrrd28/12/maso28212.html

27. Moyano JC, Marini PR, Fischman ML. 2017. Crecimiento pre-destete del ovino F1 Blackbelly x Pelibuey en condiciones de pastoreo libre en la amazonía ecuatoriana. Rev Inv Vet Peru 28: 10781083. doi: 10.15381/rivep.v28i4.13929

28. Norouzian MA. 2015. Effects of lambing season, birth type and sex on early performance of lambs. New Zeal J Agr
Res 58: 84-88. doi: 10.1080/00288233.2014.944270

29. Oliva-Hernández J, Del GarcíaOsorio IC. 2016. A note on the changes in the preweaning growth of Pelibuey lambs. Arch Med Vet 48: 293-298. doi: 10.4067/S0301-732X2016000300008

30. Petrovia MP, Petroviae VC, RužiaeMuslice D, Maksimovice N, Petrovice MM, Ilice Z, Stojkovice J. 2015a. Effect of genetic and environmental factors on the phenotype characteristics of lambs. Biotechnol Anim Husb 31: 223-233. doi: 10.2298/BAH1502223P

31. Petroviae VC, Petrovic MP, RužiaeMusliae D, Maksimoviae N, Selionova MI, Aybazov MM, Malyukova MA. 2015. Genotype, sex and interaction effect on lamb growth traits. Biotechnol Anim Husb 31: 37-44. doi: 10.2298/ BAH1501037P

32. Quintero A, Boscan, J, Palomares R, González A, Boissiere J. 1997. Efecto del sexo sobre el peso corporal a diferentes edades en corderos West-African criados en el trópico venezolano. Arch Latinoam Prod Anim 5: 426-427.

33. Ramírez-Tello JA, Torres-Hernández G, De La Cruz-Colín L, Ochoa-Cordero MA, Suárez-Espinosa J. 2013. Environmental factors influencing growth variables from birth to weaning in Hampshire lambs. Rev Mex Cienc Pecu 4: 117-125.

34. Segura J.C. 2000. Métodos de muestreo para la producción y la salud animal. México: Ed Universidad Autónoma de Yucatán. $142 \mathrm{p}$.

35. Taye M, Abebe G, Gizaw S, Lemma S, Mekoya A, Tibbo M. 2010. Growth performances of Washera sheep under smallholder management systems in Yilmanadensa and Quarit districts, Ethiopia. Trop Anim Health Pro 42: 659667. doi: 10.1007/s11250-009-9473-x

36. Teklebrhan T, Urge M, Mekasha Y, Baissa M. 2014. Pre-weaning growth performance of crossbred lambs (Dorper $\mathrm{x}$ indigenous sheep breeds) under semiintensive management in eastern 
Ethiopia. Trop Anim Health Prod 46: 455460. doi: 10.1007/s11250-013-0513-1

37. Yilmaz A, Karakus F, Bingöl M, Kaki $B$, Ser G. 2018. Effects of some factors on growth of lambs and the determination of growth curve models. Indian J Anim Res 52: 521275-1262 doi: 10.18805/ijar.B-815

38. Yilmaz M, Altin T. 2011. Growth characteristics in lambs of estrus synchronized ewes in grower conditions. Turk J Vet Anim Sci 35: 421-429. doi: 10.3906/vet-1001-226
39. Yilmaz O, Denk H, Bayram D. 2007. Effects of lambing season, sex and birth type on growth performance in Norduz lambs. Small Ruminant Res 68: 336-339. doi: 10.1016/j.smallrumres.2005.11.013

40. Zidane A, Niar A, Ababou A. 2015. Effect of some factors on lamb's growth performances of the Algerian Ouled Djellal breed. Livestock Res Rural Develop 27(7). [Internet]. Disponible en: http://www.1rrd.org/1rrd2 7/7/ zida27126.html 